\title{
Modern analyses on an historical data set: skull morphology of Italian red squirrel populations
}

\author{
Giovanni Amori', Gaetano Aloise², Luca Luiselli ${ }^{3}$ \\ I CNR - Institute of Ecosystem Studies, viale dell'Università 32, I-00185 Rome, Italy 2 Natural History Museum \\ and Botanical Garden, Calabria University 3 Environmental Studies Centre Demetra s.r.l., Rome, Italy \\ Corresponding author: Giovanni Amori (giovanni.amori@uniroma1.it)
}

Academic editor: K.M. Helgen | Received 16 January 2013 | Accepted 6 December 2013 | Published 8 January 2014

Citation: Amori G, Aloise G, Luiselli L (2014) Modern analyses on an historical data set: skull morphology of Italian red squirrel populations. ZooKeys 368: 79-89. doi: 10.3897/zookeys.368.4691

\begin{abstract}
Recent molecular evidence suggests that Sciurus vulgaris populations from Calabria (southern Italy) are distinct from those occurring in northern and central Italy. Here, we re-analyzed using multivariate and univariate techniques an historical dataset provided by Cavazza (1913), who documented measurements for the now extinct squirrel population from Campania. Both univariate and multivariate analyses confirmed that the sample from Calabria was homogenous and relatively distinct compared to the rest of the squirrel samples.
\end{abstract}

\section{Keywords}

Morphometrics, red squirrel, Italy, historical dataset

\section{Introduction}

The Eurasian red squirrel, Sciurus vulgaris Linnaeus, 1758, is characterized by great variability in fur coloration, which led to the description of more than 40 subspecies throughout its wide geographic distribution across the Eurasian continent (Corbet 1978). Currently only 17 of these subspecies are considered valid (Lurz et al. 2005), with the Italian populations being ascribed to three subspecies (Toschi 1965). These Italian subspecies are:

1) S. vulgaris fuscoater Altum, 1876 (European form occurring in the Alps and in the northern Apennines), characterized by relatively small size and a strong degree of coat-colour polymorphism both within and between populations; 
2) S. vulgaris italicus Bonaparte, 1838 (endemic to Central Italy), also characterized by relatively small size, albeit bigger than the previous subspecies. This subspecies shows some degree of coat colour polymorphism, with the dark brown morph dominant in mountainous forests at higher altitudes. The populations of the southern tip of the range are black (subspecies alpinus, sensu Costa 1839);

3) S. vulgaris meridionalis Lucifero, 1907 (endemic to the most southern Apennines), with uniform fur colour, always having black dorsal fur with grey shades on the sides, a black tail, and a contrasting white belly. It is also the largest Italian subspecies (Wauters and Martinoli 2008).

Although widespread in Italy, this species' distribution is associated with forested areas, and affected by their fragmentation (Celada et al. 1994, Wauters et al. 1994a, Wauters et al. 1994b, Wauters 1997, Hale et al. 2001). Thus, the European squirrel currently occurs in the whole of the Italian Peninsula with some distribution gaps: the species does not currently occurs in Campania, Apulia and Basilicata (cf. Wauters and Martinoli 2008). However, the squirrel was present in historical times also in the extreme northern part of Campania (i.e. Mt. Somma - Vesuvio) (Costa 1839, Trouessart 1910, Cavazza 1913), where it is now extinct (Capolongo and Caputo 1990, Maio et al. 2000).

Recent molecular data (Grill et al. 2009) revealed the presence of two main mitochondrial phylogroups: (i) a clade comprising the individuals from the region of Calabria in southern Italy belonging to the subspecies $S$. v. meridionalis, and (ii) another including the rest of the Italian populations.

Cavazza (1913) studied morphological variability of Italian populations of Sciurus vulgaris, and provided a useful set of skull measurements for squirrels collected throughout Italy. Among various populations, he analyzed specimens from an area where the species is now locally extinct (Campania), which is geographically closer to the populations of the subspecies italicus than to those of meridionalis. Cavazza's (1913) data are important for evaluating whether the extinct Campanian squirrels were more similar to those currently inhabiting Calabria, or to those typical of central Italian regions.

In this paper, we reanalyzed Cavazza's original dataset using modern statistical multivariate analyses with the aim to evaluate whether morphometric and genetic data agree with respect to patterns of geographic differentiation in Italian squirrel populations.

\section{Materials and methods}

We used the data reported in Cavazza (1913) for skull measurements of adults (Table 1). Cavazza (1913) divided specimens into the following groups: (a) Alps, (b) northern and central Italy including Latium and excluding Abruzzi, (c) southern Italy including Abruzzi and Campania, and (d) Calabria. The localities where Cavazza (1913) collected his specimens are reported in Figure 1. Unfortunately, we cannot re-measure specimens from Cavazza's (1913) paper because several of them have now become lost. Moreover, although it is possible that some of the specimens originarily measured by Cavazza 
(1913) are still available in private or public collections in Italy, unfortunately there is no labeling indication in Cavazza's paper for any of his specimens, and this fact impeded us from any further analysis of the vouchers.

Univariate measurements were log-transformed in order to achieve normality and then compared across groups by one-way Analysis of Variance (ANOVA). In this analysis, the same four groups as defined by Cavazza (1913) were used.

Specimens were divided into four Operational Taxonomic Units (hereby OTUs), according to their geographical provenance and corresponding to the Italian subspecies. These four OTUs followed exactly the subdivisions made by Cavazza (1913). We performed a cluster analysis in order to show dissimilarities among all of Cavazza's (1913) specimens in terms of their skull measurements. Skull measurements were logtransformed prior to analysis. Dendrograms were prepared using the single linkage as

Table I. Skull measurements (in mm). OTU = Operational Taxonomic Unit; A = Alps; B = North and Central Italy; $\mathrm{C}=$ Abruzzo and Campania; $\mathrm{D}=$ Calabria (from Cavazza 1913). For more details see text.

\begin{tabular}{|c|c|c|c|c|c|c|c|c|}
\hline ID & OTU & $\begin{array}{c}\text { Skull } \\
\text { length }\end{array}$ & $\begin{array}{l}\text { Skull } \\
\text { width }\end{array}$ & $\begin{array}{c}\text { Skull } \\
\text { height }\end{array}$ & $\begin{array}{c}\text { Mandible } \\
\text { length }\end{array}$ & $\begin{array}{c}\text { Interorbital } \\
\text { width }\end{array}$ & Locality & sex \\
\hline 17Alpf & A & 50.1 & 28.5 & 19.6 & 27.3 & 18.3 & AlpiCentrali & $\mathrm{f}$ \\
\hline $8 \mathrm{Apf}$ & A & 51.9 & 29.6 & 23.4 & 28.0 & 19.2 & Lanzo & $\mathrm{f}$ \\
\hline 7Alpm & A & 49.7 & 28.3 & 21.0 & 28.2 & 18.8 & Lanzo & $\mathrm{m}$ \\
\hline 12Aplf & A & 51.8 & 30.0 & 21.0 & 28.2 & 19.0 & Porlezza & $\mathrm{f}$ \\
\hline 7Alpf & A & 51.3 & 29.0 & 23.3 & 28.8 & 18.9 & Lanzo & $\mathrm{f}$ \\
\hline 3Alpf & A & 52.0 & 29.0 & 22.0 & 28.9 & 20.0 & AlpiPiem. & $\mathrm{f}$ \\
\hline 9Alpf & A & 51.2 & 29.1 & 21.3 & 28.9 & 18.4 & Porlezza & $\mathrm{f}$ \\
\hline 5Alpm & A & 49.6 & 29.0 & 20.0 & 29.0 & 19.2 & Biellese & $\mathrm{m}$ \\
\hline 13Alpm & A & 51.5 & 31.2 & 21.6 & 29.0 & 20.0 & Porlezza & $\mathrm{m}$ \\
\hline 2Alpf & A & 52.6 & 29.6 & 22.0 & 29.0 & 20.0 & AlpiPiem. & $\mathrm{f}$ \\
\hline 4Alpf & A & 51.8 & 29.0 & 21.0 & 29.0 & 19.8 & Biellese & $\mathrm{f}$ \\
\hline 15Alpf & A & 52.6 & 30.6 & 19.9 & 29.0 & 20.0 & SopraLugano & $\mathrm{f}$ \\
\hline 18Alpf & A & 51.7 & 29.8 & 21.0 & 29.0 & 18.2 & AlpiCentrali & $\mathrm{f}$ \\
\hline 25Alpf & A & 50.1 & 28.7 & 21.0 & 29.0 & 19.0 & Cadore & $\mathrm{f}$ \\
\hline 26Alpf & A & 51.2 & 29.6 & 22.0 & 29.0 & 19.4 & Cadore & $\mathrm{f}$ \\
\hline 9Alpm & A & 51.8 & 30.6 & 21.0 & 29.1 & 19.5 & Lanzo & $\mathrm{m}$ \\
\hline 16Alpf & A & 55.0 & 31.2 & 21.0 & 29.1 & 18.6 & AlpiCentrali & $\mathrm{f}$ \\
\hline 1Alpf & A & 53.0 & 30.8 & 22.0 & 29.2 & 21.0 & AlpiPiem. & $\mathrm{f}$ \\
\hline 5Alpf & A & 50.8 & 28.9 & 22.6 & 29.2 & 18.7 & Lanzo & $\mathrm{f}$ \\
\hline 13Alpf & A & 55.9 & 31.0 & 22.6 & 29.2 & 20.2 & Buggiolo & $\mathrm{f}$ \\
\hline 21Alpf & A & 53.0 & 30.0 & 21.2 & 29.2 & 19.0 & AlpiCentrali & $\mathrm{f}$ \\
\hline 2Alpm & $\mathrm{A}$ & 57.3 & 32.0 & 21.0 & 29.3 & 20.0 & AlpiPiem. & $\mathrm{m}$ \\
\hline 10Alpm & $\mathrm{A}$ & 52.2 & 31.0 & 21.0 & 29.3 & 20.0 & Lanzo & $\mathrm{m}$ \\
\hline $14 \mathrm{Alpm}$ & A & 49.9 & 27.8 & 20.8 & 29.3 & 18.3 & Porlezza & $\mathrm{m}$ \\
\hline 6Alpf & $\mathrm{A}$ & 52.0 & 29.8 & 23.0 & 29.3 & 19.1 & Lanzo & $\mathrm{f}$ \\
\hline 14Alpf & $\mathrm{A}$ & 52.6 & 30.3 & 21.8 & 29.3 & 21.0 & SopraLugano & $\mathrm{f}$ \\
\hline 20Alpf & A & 51.8 & 29.9 & 21.2 & 29.3 & 19.0 & AlpiCentrali & $\mathrm{f}$ \\
\hline 19Alpf & $\mathrm{A}$ & 52.0 & 30.0 & 21.3 & 29.4 & 19.5 & AlpiCentrali & $\mathrm{f}$ \\
\hline 12Alpm & A & 52.0 & 30.2 & 21.6 & 29.5 & 20.0 & Lanzo & $\mathrm{m}$ \\
\hline
\end{tabular}




\begin{tabular}{|c|c|c|c|c|c|c|c|c|}
\hline ID & OTU & $\begin{array}{r}\text { Skull } \\
\text { length }\end{array}$ & $\begin{array}{l}\text { Skull } \\
\text { width }\end{array}$ & $\begin{array}{c}\text { Skull } \\
\text { height }\end{array}$ & $\begin{array}{c}\text { Mandible } \\
\text { length }\end{array}$ & $\begin{array}{c}\text { Interorbital } \\
\text { width }\end{array}$ & Locality & sex \\
\hline 3Alpm & A & 50.0 & 29.2 & 20.4 & 29.6 & 19.2 & AlpiPiem. & $\mathrm{m}$ \\
\hline 6Alpm & $\mathrm{A}$ & 52.7 & 30.3 & 22.2 & 29.8 & 19.8 & Lanzo & $\mathrm{m}$ \\
\hline $1 \mathrm{Alpm}$ & $\mathrm{A}$ & 53.0 & 32.2 & 21.2 & 30.0 & 20.7 & AlpiPiem. & $\mathrm{m}$ \\
\hline 18Alpm & A & 52.8 & 32.0 & 21.0 & 30.0 & 20.0 & SopraLugano & $\mathrm{m}$ \\
\hline 4Alpm & A & 53.0 & 33.0 & 21.3 & 31.0 & 20.0 & Biellese & $\mathrm{m}$ \\
\hline 26Alpm & $\mathrm{B}$ & 51.5 & 30.3 & 21.0 & 28.9 & 19.3 & Cadore & $\mathrm{m}$ \\
\hline 25Alpm & $\mathrm{B}$ & 51.6 & 30.8 & 20.8 & 29.0 & 19.0 & Cadore & $\mathrm{m}$ \\
\hline 1Lomm & $\mathrm{B}$ & 52.2 & 29.4 & 22.6 & 29.0 & 18.8 & Lombardia & $\mathrm{m}$ \\
\hline 2Emim & B & 52.6 & 29.0 & 22.2 & 29.0 & 19.4 & Emilia & $\mathrm{m}$ \\
\hline 3Emif & B & 50.1 & 29.0 & 21.8 & 29.0 & 18.6 & Emilia & $\mathrm{f}$ \\
\hline 9Tosf & B & 53.2 & 30.2 & 22.1 & 29.0 & 18.8 & Toscana & $\mathrm{f}$ \\
\hline 10Tosf & $\mathrm{B}$ & 52.8 & 29.7 & 22.9 & 29.0 & 18.0 & Toscana & $\mathrm{f}$ \\
\hline 4Emif & $\mathrm{B}$ & 51.1 & 29.3 & 22.0 & 29.1 & 18.9 & Emilia & $\mathrm{f}$ \\
\hline 5Emif & $\mathrm{B}$ & 52.0 & 29.0 & 22.0 & 29.1 & 18.7 & Emilia & $\mathrm{f}$ \\
\hline 11Tosm & $\mathrm{B}$ & 52.0 & 30.0 & 22.0 & 29.2 & 18.6 & Toscana & $\mathrm{m}$ \\
\hline 1Ligf & B & 51.2 & 28.3 & 22.0 & 29.2 & 18.3 & Liguria & $\mathrm{f}$ \\
\hline 6Emif & B & 52.2 & 29.1 & 22.3 & 29.3 & 18.6 & Emilia & $\mathrm{f}$ \\
\hline 7Emim & $\mathrm{B}$ & 52.7 & 30.1 & 22.3 & 29.5 & 19.0 & Emilia & $\mathrm{m}$ \\
\hline 3Emim & $\mathrm{B}$ & 53.7 & 29.6 & 23.0 & 29.6 & 19.9 & Emilia & $\mathrm{m}$ \\
\hline 8Emim & B & 52.7 & 30.2 & 22.2 & 29.6 & 18.9 & Emilia & $\mathrm{m}$ \\
\hline 10Tosm & $\mathrm{B}$ & 53.2 & 30.1 & 22.0 & 29.6 & 19.0 & Toscana & $\mathrm{m}$ \\
\hline $9 \mathrm{mim}$ & $\mathrm{B}$ & 52.7 & 30.3 & 22.2 & 29.8 & 19.0 & Emilia & $\mathrm{m}$ \\
\hline $1 \mathrm{Alpm}$ & $\mathrm{B}$ & 52.9 & 30.1 & 21.3 & 30.0 & 19.8 & AlpiCentrali & $\mathrm{m}$ \\
\hline 20Alpm & $\mathrm{B}$ & 52.3 & 30.1 & 20.6 & 30.0 & 18.9 & AlpiCentrali & $\mathrm{m}$ \\
\hline 22Alpm & B & 50.0 & 31.0 & 22.0 & 30.0 & 18.8 & AlpiCentrali & $\mathrm{m}$ \\
\hline 6Emim & $\mathrm{B}$ & 53.5 & 34.3 & 22.1 & 30.0 & 18.0 & Emilia & $\mathrm{m}$ \\
\hline 12Tosf & $\mathrm{B}$ & 53.0 & 30.8 & 22.9 & 30.0 & 18.3 & Toscana & $\mathrm{f}$ \\
\hline 13Tosf & $\mathrm{B}$ & 52.3 & 30.3 & 22.8 & 30.0 & 18.7 & Toscana & $\mathrm{f}$ \\
\hline 18Tosm & $\mathrm{B}$ & 52.2 & 31.0 & 22.3 & 30.2 & 18.7 & Toscana & $\mathrm{m}$ \\
\hline 21Alpm & $\mathrm{B}$ & 53.1 & 32.0 & 21.2 & 30.3 & 19.7 & AlpiCentrali & $\mathrm{m}$ \\
\hline 17Tosm & $\mathrm{B}$ & 53.0 & 32.0 & 22.0 & 30.3 & 18.0 & Toscana & $\mathrm{m}$ \\
\hline 13Tosm & $\mathrm{B}$ & 52.0 & 30.0 & 20.8 & 30.6 & 18.2 & Toscana & $\mathrm{m}$ \\
\hline 11Tosf & $\mathrm{B}$ & 52.0 & 31.5 & 23.0 & 31.0 & 18.7 & Toscana & $\mathrm{f}$ \\
\hline 12Tosm & $\mathrm{B}$ & 55.0 & 31.9 & 21.0 & 31.2 & 19.0 & Toscana & $\mathrm{m}$ \\
\hline 3Napf & $\mathrm{C}$ & 52.3 & 29.7 & 24.1 & 28.9 & 18.6 & Napoletano & $\mathrm{f}$ \\
\hline 4Napf & $\mathrm{C}$ & 54.6 & 29.9 & 25.0 & 29.0 & 19.0 & Napoletano & $\mathrm{f}$ \\
\hline $3 \mathrm{Napm}$ & $\mathrm{C}$ & 52.8 & 28.9 & 22.9 & 29.2 & 19.0 & Napoletano & $\mathrm{m}$ \\
\hline 2Napf & $\mathrm{C}$ & 54.3 & 29.8 & 24.9 & 29.4 & 18.9 & Napoletano & $\mathrm{f}$ \\
\hline 4Napm & $\mathrm{C}$ & 55.0 & 29.8 & 22.8 & 29.5 & 19.6 & Napoletano & $\mathrm{m}$ \\
\hline 2Napm & $\mathrm{C}$ & 55.2 & 31.3 & 24.0 & 30.0 & 20.0 & Napoletano & $\mathrm{m}$ \\
\hline 2Calf & $\mathrm{D}$ & 56.3 & 33.6 & 22.7 & 31.8 & 19.1 & Calabria & $\mathrm{f}$ \\
\hline 3Calm & $\mathrm{D}$ & 56.0 & 33.9 & 22.4 & 32.2 & 19.0 & Calabria & $\mathrm{f}$ \\
\hline 1Calm & $\mathrm{D}$ & 56.0 & 33.5 & 22.6 & 33.9 & 20.7 & Calabria & $\mathrm{f}$ \\
\hline 1Calf & $\mathrm{D}$ & 57.2 & 33.4 & 22.8 & 33.9 & 19.2 & Calabria & $\mathrm{f}$ \\
\hline 2Calm & $\mathrm{D}$ & 54.5 & 32.9 & 22.3 & 34.1 & 20.2 & Calabria & $\mathrm{f}$ \\
\hline
\end{tabular}




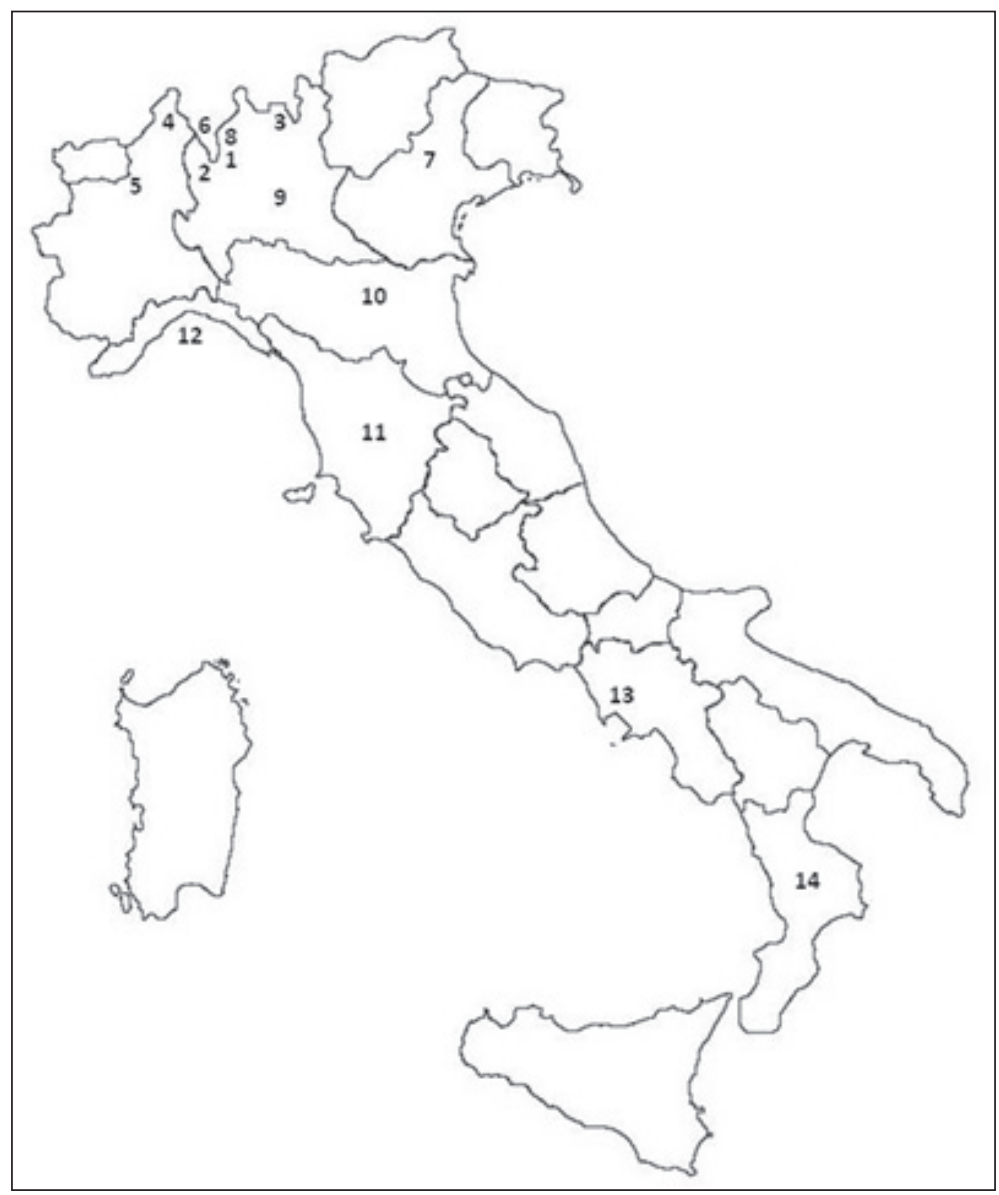

Figure I. Map of Italy showing the localities where squirrels were collected according to Cavazza (1913). I Porlezza 2 Lanzo 3 Central Alps 4 Alpi Piemontesi 5 Biellese 6 Lugano 7 Bassano del Grappa 8 Buggiolo 9 Lombardia 10 Emilia I I Tuscany $\mathbf{2}$ Liguria $\mathbf{3}$ Neapolitan (Campania) I 4 Calabria.

the algorithm, with Euclidean distances. This method was used because it provided the highest cophenetic index. In the single linkage (nearest neighbour), the clusters are joined based on the smallest distance between the two groups. Branch support was calculated with 10,000 bootstrap replicates. We also used neighbour joining clustering (Saitou and Nei 1987), which is an alternative method for hierarchical cluster analysis. In contrast with ultrametric methods (like the Unweighted Pair Group Method with Arithmetic Mean, UPGMA), two branches from the same internal node do not need to have equal branch lengths. A phylogram (unrooted dendrogram with proportional branch lengths) is given in this paper.

We studied the dispersion of specimens in multivariate space with Principal Components Analysis (PCA) using the covariance matrix (Davis 1986, Harper 1999) (PC1 scores serve as a proxy for size, while the other PCs capture shape variation). 
Table 2. Mean and dispersion measures of the five skull variables analyzed in this study (original dataset from Cavazza (1913), for all sampled specimens pooled together.

\begin{tabular}{l|c|c}
\hline & Mean (S.D.) & Range \\
\hline Skull length & $52.61(1.70)$ & $49.6-57.3$ \\
\hline Skull width & $30.37(1.41)$ & $27.8-34.3$ \\
\hline Skull height & $21.92(1.04)$ & $19.6-25.0$ \\
\hline Mandible length & $29.63(1.18)$ & $27.3-34.1$ \\
\hline Interorbital length & $19.21(0.70)$ & $18.0-21.0$ \\
\hline
\end{tabular}

Table 3. Mean and dispersion measures of the five skull variables analyzed in this study (original dataset from Cavazza (1913), with all sampled specimens divided by OTU. Symbols: A = Alps; B = North and Central Italy; C = Abruzzo and Campania; D = Calabria.

\begin{tabular}{|c|c|c|}
\hline & Mean & SD \\
\hline \multicolumn{3}{|c|}{$\mathrm{A}(\mathrm{n}=34)$} \\
\hline Skull length & 52.05 & 1.64 \\
\hline Skull width & 30.03 & 1.19 \\
\hline Skull height & 21.42 & 0.88 \\
\hline Mandible length & 29.12 & 0.62 \\
\hline Interorbital length & 19.46 & 0.74 \\
\hline \multicolumn{3}{|c|}{$\mathrm{B}(\mathrm{n}=29)$} \\
\hline Skull length & 52.37 & 1.02 \\
\hline Skull width & 30.32 & 1.20 \\
\hline Skull height & 21.97 & 0.68 \\
\hline Mandible length & 29.67 & 0.63 \\
\hline Interorbital length & 18.22 & 0.49 \\
\hline \multicolumn{3}{|c|}{$C(n=6)$} \\
\hline Skull length & 54.03 & 1.20 \\
\hline Skull width & 29.90 & 0.77 \\
\hline Skull height & 23.95 & 0.94 \\
\hline Mandible length & 29.33 & 0.39 \\
\hline Interorbital length & 19.18 & 0.51 \\
\hline \multicolumn{3}{|c|}{$\mathrm{D}(\mathrm{n}=5)$} \\
\hline Skull length & 56.00 & 0.97 \\
\hline Skull width & 33.46 & 0.36 \\
\hline Skull height & 22.56 & 0.20 \\
\hline Mandible length & 33.18 & 1.08 \\
\hline Interorbital length & 19.64 & 0.76 \\
\hline
\end{tabular}

\section{Results}

The original dataset reported by Cavazza (1913) is summarized in Table 1. Mean and standard deviations for each measurement considered are reported in Table 2 with all specimens pooled, and in Table 3 with samples divided into OTUs. Using the same categories as in Cavazza (1913), there were among-group statistical differences for skull 


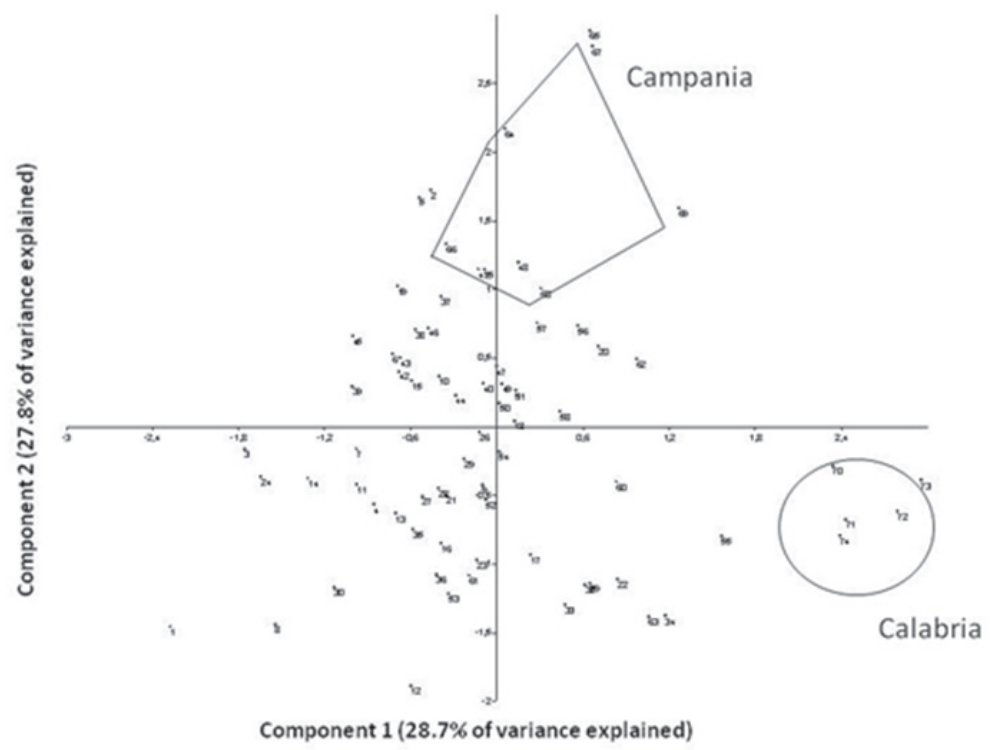

Figure 2. PCA of skull measurements (VARIMAX rotation applied) based on Cavazza's (1913) dataset. Eigenvalues: component $1=2.559$; component $2=1.099$.

length (one-way ANOVA $\left.\mathrm{F}_{3,70}=14.76, \mathrm{P}<0.00001\right)$, skull width $\left(\mathrm{F}_{3,70}=13.50, \mathrm{P}<\right.$ $0.00001)$, skull height $\left(\mathrm{F}_{3,70}=18.93, \mathrm{P}<0.00001\right)$, and mandible length $\left(\mathrm{F}_{3,70}=56.83\right.$, $\mathrm{P}<0.00001)$, but not for interorbital length $\left(\mathrm{F}_{3,70}=1.92, \mathrm{P}<0.133\right)$. Post-hoc Tukey HSD tests revealed that Calabria specimens differed significantly from every other group for mandible length (all $\mathrm{P}<0.01$ ), and for skull width (all $\mathrm{P}<0.001$ ). For skull length, Calabria specimens differed from Alpine and central Italian specimens (all $\mathrm{P}<0.01$ ) but not from Campania specimens $(\mathrm{P}=0.088)$. For skull height, they differed from Campania $(\mathrm{P}=0.024)$ and Alpine specimens $(\mathrm{P}=0.018)$ but not from central Italian specimens $(\mathrm{P}=0.43)$. Principal component scores indicated that there were significant statistical shape differences among the four populational groups (one-way ANOVA: $\mathrm{F}_{3,70}=30.362$, $\mathrm{P}<0.0001$ ), and a Tukey HSD post-hoc test revealed that (i) the Calabria population differed significantly from all the others (at least, $\mathrm{P}<0.000154$ ), (ii) the Campania population significantly differed, other than from Calabria specimens, also from Alpine specimens $(P=0.022)$ but not from central Italian specimens $(P=0.470)$.

Both sets of multivariate analyses revealed that the sample from Calabria was homogenous and relatively distinct compared to the rest of the squirrel samples (Figures 2 and 3). In the PCA (variance explained by the first two axes: 56.5\%; with axis 1 explaining $28.7 \%$ and axis 2 explaining $27.8 \%$ of the total variance; see Table 4 for the loadings) there was a trend suggesting clinal variation from the Alps to Campania, with Calabria specimens, while distinct, being more similar to those of Campania than to those of northern Italy (Figure 2). The Campania group showed less variance (Levene's test; $\mathrm{F}=6.67, \mathrm{P}<0.03$ ) compared to the rest of the central and northern Italian samples in the PCA than in the neighbor joining analysis (Figure 3). 
Table 4. Loadings of the PCA as in Figure 2.

\begin{tabular}{l|c|c}
\hline & Component 1 & Component 2 \\
\hline Skull length & 0.876 & 0.154 \\
\hline Skull width & 0.882 & -0.159 \\
\hline Skull height & 0.341 & 0.836 \\
\hline Mandible length & 0.842 & -0.034 \\
\hline Interorbital length & 0.432 & -0.684 \\
\hline
\end{tabular}

\section{Discussion}

Both multivariate and univariate tests identified some morphometric differentiation among different squirrel populations that were previously highlighted by the molecular results of Grill et al. (2009). That is: the populations from Calabria differed from the others morphologically (this study) and genetically (D-Loop: Mean genetic distance between groups: 6\%, within group: 2\%; see Grill et al. 2009). Our analyses also suggest that the currently extinct population from Campania belonged to a central Italian grouping. It may be that patterns of craniometric variation in Italian red squirrels represent a clinal size trend within a formerly contiguous population once occurring from the Alps south to Campania, and, with expectations fitting Bergmann's rule (e.g., Freckleton et al. 2003; Blackburn and Hawkins 2004).

On the other hand, Calabria specimens do appear to be quite distinct from the rest of the Italian squirrels in size (Figure 2), though we note that our analyses involve quite small sample sizes (Cardini and Elton 2007). Notably, Calabria populations occur mainly at relatively high altitudes, closely linked to that of extensive high-altitude mixed forest dominated by the native Calabrian black pine Pinus laricio (Cagnin et al. 2000, Rima et al. 2010) and they are characterized both by large size and monomorphic color fur. Overall, our study could neither substantiate nor reject the hypothesis that Sciurus vulgaris meridionalis is a full species, as previously suggested by Gippoliti (2013). However, some morphological differentiation is certainly evident also with respect to the Campania extinct population (this study), and remarkable genetic differences are found between Calabria populations and all the remaining European populations (Grill et al. 2009). Indeed, the majority of individuals analyzed by Grill et al. (2009) formed one monophyletic clade without particular differentiation, whereas Calabrian squirrels were clearly separate. The Calabrian lineage appears to have experienced a different history from the rest of European squirrels probably due to the fact that it became isolated after glaciations and never reconnected to Central Italian populations (Grill et al. 2009). It should be stressed, however, that the sample sizes available for Campania and Calabria were too small to make any firm conclusions.

Our approach in this paper highlights the lasting value of historical publications on biodiversity, especially when they present data on populations which are now extinct. These often overlooked publications - such as Cavazza's, published in Italian in a regional journal - can be important sources of data that can be re-analysed, for renewed insight, using modern statistical tools. 


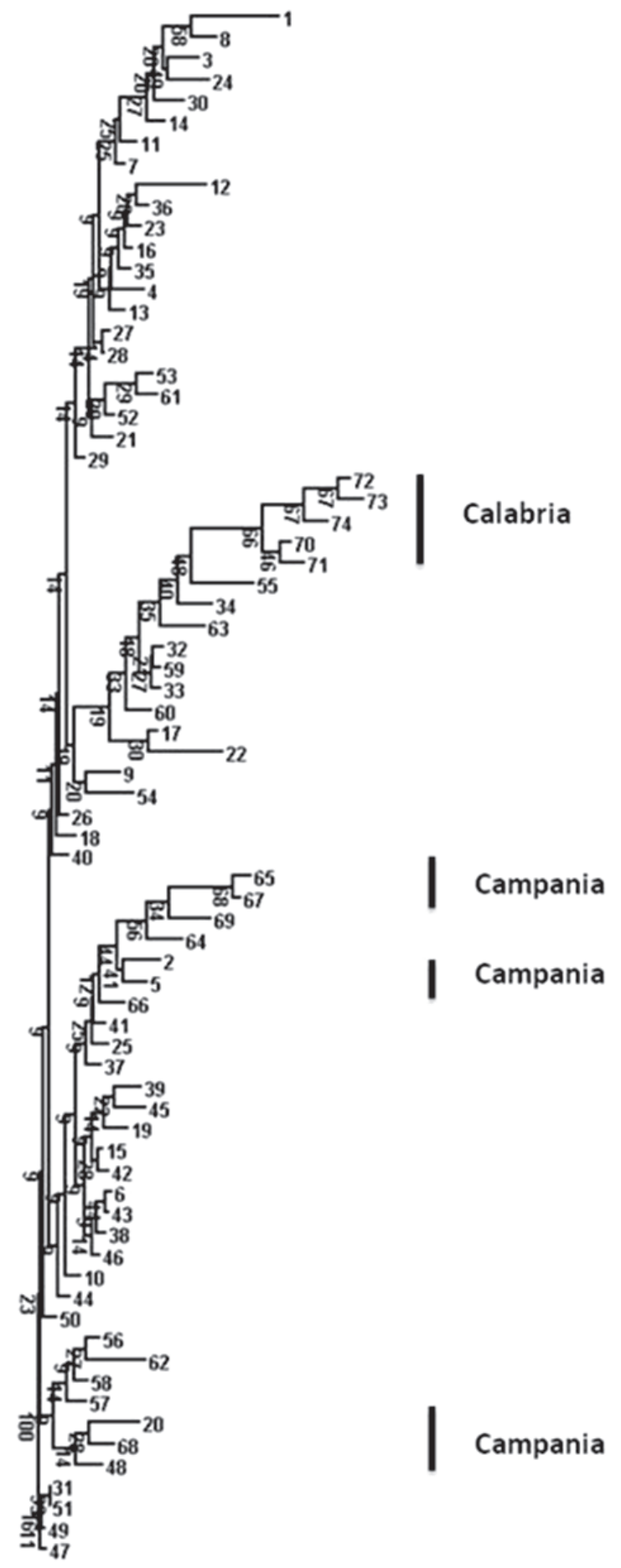

Figure 3. Neighbor joining dendrogram of skull measurements (with 10,000 bootstraps) based on Cavazza's (1913) dataset. 


\section{Acknowledgements}

This paper was edited by Russell L. Burke (Hofstra University, New York). We thank A. Cardini and an anonymous referee for very helpful comments on the submitted draft.

\section{References}

Blackburn TM, Hawkins BA (2004) Bergmann's rule and the mammal fauna of northern North America. Ecography 27: 715-724. doi: 10.1111/j.0906-7590.2004.03999.x

Cagnin M, Aloise G, Fiore F, Oriolo V, Wauters L (2000) Habitat use and population density of the Calabrian squirrel Sciurus vulgaris meridionalis Lucifero, 1907 in the Sila Grande mountain range (Calabria, Italy). Italian Journal of Zoology 67: 81-87. doi: $10.1080 / 11250000009356299$

Capolongo D, Caputo V (1990) Mammals of the Partenio Mountains (Campanian Apennines, southern Italy). Vie Milieu 40: 156-159.

Cardini A, Elton S (2007) Sample size and sampling error in geometric morphometric studies of size and shape. Zoomorphology 126: 121-134. doi: 10.1007/s00435-007-0036-2

Cavazza F (1913) Studio intorno alla variabilità dello Sciurus vulgaris in Italia. Atti della Regia Accademia dei Lincei, Classe Science Fisiche, Matematiche e Naturali, IX, Ser. 5: 504-593.

Celada C, Bogliani G, Gariboldi A, Maracci A (1994) Occupancy of isolated woodlots by the red squirrel Sciurus vulgaris L. in Italy. Biological Conservation 69: 177-183. doi: 10.1016/0006-3207(94)90057-4

Corbet GB (1978) The Mammals of the Palaearctic region: a taxonomic review. British Museum (Natural History), London, UK.

Costa OG (1839) Fauna del Regno di Napoli, ossia enumerazione di tutti gli animali che abitano le diverse regioni di questo Regno e le acque le bagnano, contenente la descrizione de' nuovi o poco esattamente conosciuti. Stamperia Azzolino e Compagno, Napoli, Italy.

Davis JC (1986) Statistics and Data Analysis in Geology. John Wiley and Sons, New York, USA. Freckleton RP, Harvey PH, Pagel M (2003) Bergmann's rule and body size in mammals. American Naturalist 161: 821-825. doi: 10.1086/374346

Gippoliti S (2013) Checklist delle specie dei mammiferi italiani (esclusi Mysticeti e Odontoceti): contributo per la conservazione della biodiversità. Bolletino del Museo Civico di Storia Naturasle di Verona (Botanica e Zoologia) 37: 7-28.

Grill A, Amori G, Aloise G, Lisi I, Tosi G, Wauters L, Randi E (2009) Molecular phylogeography of European Sciurus vulgaris: refuge within refugia? Molecular Ecology 18: 2687-2699. doi: 10.1111/j.1365-294X.2009.04215.x

Hale ML, Lurz PWW, Shirley MDF, Rushton S, Fuller RM, Wolff K (2001) Impact of landscape mamagement on the genetic structure of red squirrel populations. Science 293: 2246-2248. doi: 10.1126/science.1062574

Harper DAT (Ed) (1999) Numerical Palaeobiology. John Wiley and Sons, New York, USA. Lurz PWW, Gurnell J, Magris L (2005) Sciurus vulgaris. Mammalian Species 769: 1-10. doi: 10.1644/1545-1410(2005)769[0001:SV]2.0.CO;2 
Maio N, Aprea G, D’Amora G, Picariello O (2000) La teriofauna del Parco Nazionale del Vesuvio ed aree limitrofe. In: Picariello O, Di Fusco N, Fraissinet M (Eds) Elementi di biodiversità del Parco Nazionale del Vesuvio. Ente Parco Nazionale del Vesuvio, Napoli, Italy.

Rima P, Cagnin M, Aloise G, Preatoni D, Wauters LA (2010) Scale-dependent environmental variables affecting red squirrel (Sciurus vulgaris meridionalis) distribution. Italian Journal of Zoology 77: 92-101. doi: 10.1080/11250000902766926

Saitou N, Nei M (1987) The neighbor-joining method: a new method for reconstructing phylogenetic trees. Molecular Biology and Evolution 4: 406-425.

Toschi A (1965) Mammalia. Lagomorpha, Rodentia, Carnivora, Artiodactyla, Cetacea. Fauna d'Italia. Vol. VII. Calderini Ed., Bologna, Italy.

Trouessart EL (1910) Faune des Mammiféres d'Europe. Friedländer \& Sohn, Berlin, Germany.

Wauters LA (1997) The ecology of red squirrel in fragmented habitats: a review. In: Gurnell J, Lurz PWW (Eds) The conservation of red squirrel, Sciurus vulgaris L. Peoples Trust of Endangered Species, London, UK, 5-12.

Wauters LA, Casale P, Dhondt AA (1994a) Space use and dispersal in red squirrel in fragmented habitats. Oikos 69: 140-146. doi: 10.2307/3545294

Wauters LA, Hutchinson Y, Parkin DT, Dhondt AA (1994b) The effects of habitat fragmentation on demography and on the loss of genetic variation in the red squirrel. Proceeedings of the Royal Society, London, B 255: 107-111.

Wauters LA, Martinoli A (2008) Sciurus vulgaris Linnaeus, 1758. In: Amori G, Contoli L, Nappi A (Eds) Mammalia II, Erinaceomorpha, Soricomorpha, Lagomorpha, Rodentia. Fauna d'Italia XLIV: 349-360. 